\title{
An in vitro hand simulator for simultaneous control of hand and wrist movements
}

\author{
Reza Sharif Razavian, Daniel Dreyfuss, Mai Katakura, Maxim D. Horwitz, Angela E. Kedgley
}

\begin{abstract}
A human hand is a complex biomechanical system, in which bones, ligaments, and musculotendon units dynamically interact to produce seemingly simple motions. A new physiological hand simulator has been developed, in which electromechanical actuators apply load to the tendons of extrinsic hand and wrist muscles to recreate movements in cadaveric specimens in a biofidelic way. This novel simulator simultaneously and independently controls the movements of the wrist (flexion/extension and radio-ulnar deviation) and flexion/extension of the fingers and thumb. Control of these four degrees of freedom (DOF) is made possible by actuating eleven extrinsic muscles of the hand. The coupled dynamics of the wrist, fingers, and thumb, and the over-actuated nature of the human musculoskeletal system make feedback control of hand movements challenging. Two control algorithms were developed and tested. The optimal controller relies on an optimization algorithm to calculate the required tendon tensions using the collective error in all DOFs, and the action-based controller loads the tendons solely based on their actions on the controlled DOFs (e.g., activating all flexors if a flexing moment is required). Both controllers resulted in hand movements with small errors from the reference trajectories $\left(<3.4^{\circ}\right)$; however, the optimal controller achieved this with $16 \%$ lower total force. Owing to its simpler structure, the actionbased controller was extended to enable feedback control of grip force. This simulator has been shown to be a highly repeatable tool $\left(<0.25 \mathbf{N}\right.$ and $<0.2^{\circ}$ variations in force and kinematics, respectively) for in vitro analyses of human hand biomechanics.
\end{abstract}

Index Terms-In-vitro hand simulator, tendon driven, hand control, grip control, real-time optimal control.

\section{INTRODUCTION}

In vitro testing is the analysis of a biological system outside its living form. In the biomechanical analysis of the human musculoskeletal system, in vitro testing can provide valuable information that is otherwise very difficult or impossible to obtain in vivo. An important challenge in in vitro analyses of complex joints is the recreation of dynamic motions in a biofidelic way. To address this issue, many joint simulators have been developed. In a physiological joint simulator, the joint under study is moved by pulling on its agonist and antagonist tendons to create joint kinematics mimicking the natural movement conditions. When the anatomy of the joint is intact, this method gives the most accurate representation of the joint kinematics during functional movements.

The biomechanics of the human hand is notoriously complex. With so many components, it is difficult to characterize

R. Sharif Razavian (corresponding author, email: r.sharifrazavian@northeastern.edu), M. Katakuta, and A. E. Kedgley are with the Department of Bioengineering, Imperial College London, London, UK. D. Dreyfuss and M. D. Horwitz are with Chelsea and Westminster Hospital, London, UK. M. Katakuta is also with Tokyo Medical and Dental University, Tokyo, Japan. in vivo, and to model in silico (i.e. computer simulations). Therefore, in vitro simulators have been developed for the fingers and the wrist [1]-[4] with applications ranging from estimating tendon moment arms [5] to predicting the outcome of surgical interventions [6]. There are 16 independent muscles acting simultaneously on the wrist. However, existing active wrist simulators [1]-[3], [7] actuate only four to six primary wrist muscles. All of these simulators neglect the importance of the extrinsic hand muscles (whose tendons originate in the forearm, cross the wrist, and insert into the phalanges) on the kinematics and kinetics of the wrist. Due to the linked action of the extrinsic muscles on digits and the wrist [8], [9], it is not trivial to dismiss them. Flexor digitorum profundus has the largest physiological cross-section area among all the wrist muscles [10], and creates significant flexion moments around the wrist when activated. Therefore, faithful in vitro replication of natural wrist movements requires the inclusion of extrinsic muscles in the physiological simulator.

A crucial consideration in a physiological simulator is how to apply load to the tendons to obtain valid results. Various methods have been proposed in the literature to tension the tendons. In the simplest form, passive forces (e.g. using dead weights or springs) are applied to the tendons and the joint is moved manually [11]-[14]. For the utmost flexibility and biofidelity, an active controller is needed to reliably drive the hand through the reference motions. In active simulators, the tendons are loaded using controllable electromechanical, hydraulic, or pneumatic actuators. Both feed-forward [3] and feedback [1], [2], [7] control architectures have been used for wrist motion creation. The redundancy in the human musculature poses a challenge in calculating muscle forces to move the joint. The control of a hand simulator is further challenging, due to the coupled dynamics of the hand, where actuation of one degree of freedom (DOF) affects another.

To address these challenges in the feedback control of a hand simulator, inspirations can be taken from the the musculoskeletal simulation literature. A few control models are presented that have the potential for feedback motion control of complex biomechanical systems. Among the published approaches are the controllers based on artificial neural networks [15], [16], advanced optimal controllers [17]-[19], and controllers based on muscle synergies and task-space [20], [21]. Most of these controllers require detailed knowledge about the dynamics of the system, or rely on extensive training data. For a control algorithm to be useful in an in vitro simulator, it must be fast enough to be implementable in realtime. Additionally, it must be able to handle the variability that exists between specimens without requiring extensive training 


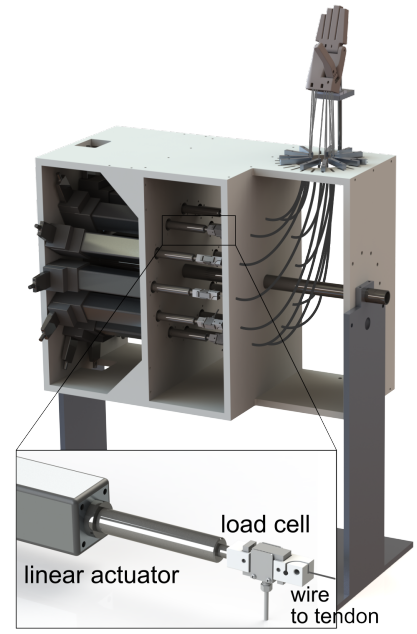

(a)

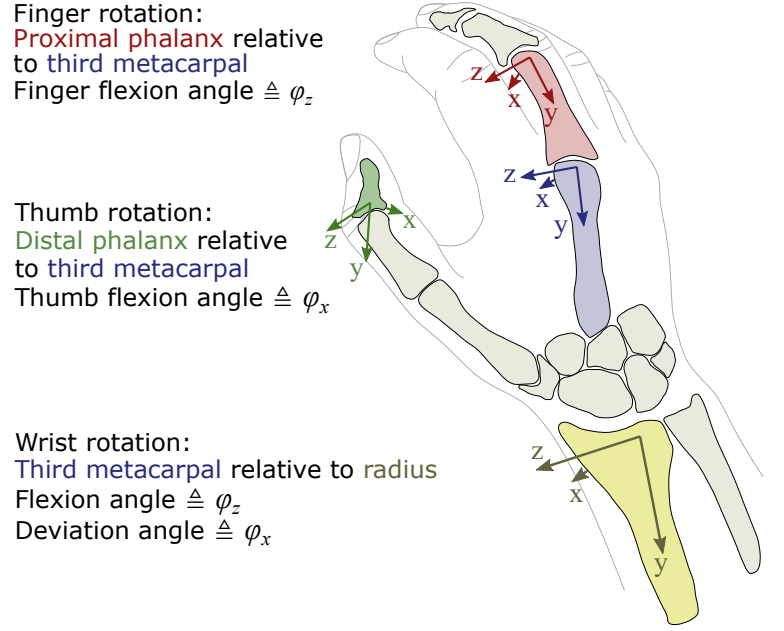

(b)

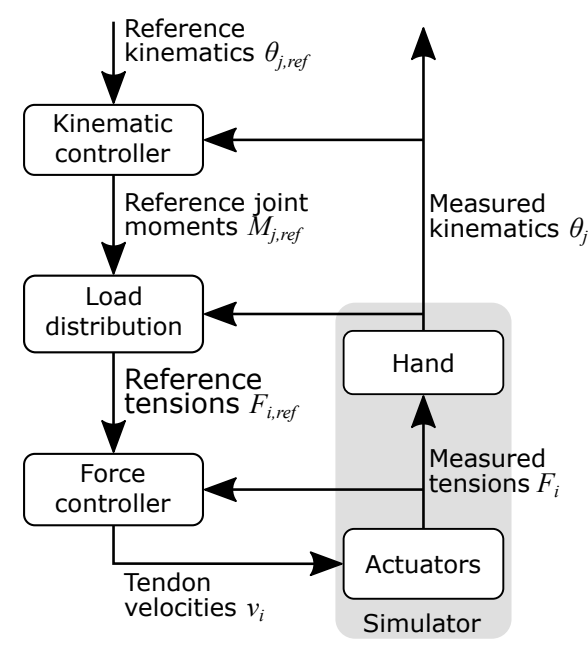

(c)

Fig. 1. (a) The hardware that provided actuation of eleven tendons. A load cell (inset) attached to each linear actuator unit measured tendon tension. Stainless steel wire ropes transferred the load from the linear actuators to the tendons. (b) The coordinate frames defined for individual hand segments. ZXY Euler sequence was used to define the relative segment angles. Finger flexion was equal to the metacarpophalangeal joint angle; thumb flexion was the total of carpometacarpal, metacarpophalangeal, and interphalangeal joint angles. (c) The schematic of the multilayer simulator controller. Two separate algorithms were developed for the load distribution layer: an optimal controller, and an action-based one. The supplementary video shows the simulator in action.

data. These concerns necessitate modifications to the available control models.

In this paper, we present the development of a new in vitro hand simulator. We had three objectives with this design.

(1) Engage all the major extrinsic hand and wrist muscles for more accurate in vitro simulations; the inclusion of all extrinsic muscles also allowed us to animate the fingers and thumb concurrently with the wrist $-\mathrm{a}$ feature that had not been implemented in any other simulator. (2) Maintain similar or better repeatability and accuracy of movements as compared to previous active simulators. (3) Design the motion controller to be bio-fidelic, mimicking human's natural movement control.

\section{METHODS: DESIGN AND CONTROL OF THE SIMULATOR}

\section{A. Simulator hardware}

Eleven electromechanical linear actuators apply tension to the extrinsic tendons of the hand (Fig. 1a). Each actuator is composed of a rotary servo motor (Moog SmartMotor SM23165d, California, USA) and a ball-screw (Mech Line 32, $100 \mathrm{~mm}$ stroke, AutomationWare S.r.l., Martellago, Italy), which transforms the rotation of the motor shaft to a linear motion. The $100 \mathrm{~mm}$ range of the linear actuators is larger than the maximum tendon excursion in a human hand. Therefore, the simulator can move all the joints in the hand in their full range of motion. A single-axis load cell (DBBSMM-25kg, Applied Measurements Ltd, Aldermaston, UK) attached to the end-point of each linear actuator measures the tension applied to the tendons (Fig. 1a.)

The fixture that holds the specimen is allowed rotation about its central axis to facilitate studying the hand in different orientations. Stainless steel wire ropes $(1 \mathrm{~mm}$ diameter $)$ are affixed to the end of the load cells to transfer the tension to the tendons. The wires are redirected from the actuator units to the tendons using incompressible Bowden cable sheaths. The Bowden cables guide the steel wire ropes to keep the tendons' lines of action as anatomical as possible. Detailed illustrations of the setup are provided as the supplementary materials. See the supplementary video for the simulator in action.

\section{B. Simulator control algorithm}

To measure the kinematics of the hand in real-time, an optical motion capture system with 8 cameras (Oqus 500, Qualisys AB, Gothenburg, Sweden) and retro-reflective markers was used. Marker clusters were rigidly affixed to the radius (forearm cluster), third metacarpal (hand cluster), proximal phalanx of the third digit (finger cluster), and distal phalanx of thumb (thumb cluster) to track 3-dimensional orientation of the segments. Body-fixed local coordinate frames (Fig. 1b) were defined after digitizing bony landmarks according to the recommendations by the International Society of Biomechanics [22]. The joint angles were calculated as the Euler angles (ZXY sequence) between the body-fixed local coordinate systems. The wrist angles were calculated as the relative rotation between the forearm and hand coordinate frames, and the finger and thumb rotations were defined as the rotations of their coordinate frames relative to that of the hand. Consequently, the four measured joint angles were: wrist flexion/extension $\left(\theta_{1}\right)$; wrist radial/ulnar deviation $\left(\theta_{2}\right)$; finger flexion $\left(\theta_{3}\right.$ which equals the metacarpophalangeal joint angle); and thumb flexion $\left(\theta_{4}\right.$, which equals the summation of carpometacarpal, metacarpophalangeal, and interphalangeal joint angles). These kinematic data were streamed in realtime to LabVIEW (National Instruments, Austin, TX, USA), in which all the data acquisition and control processes took place.

1) Kinematic controller: Real-time feedback control of the hand kinematics was achieved by modulating the tensions applied to the tendons. The high-level kinematic controller (Fig. 1c) includes multiple (one for each DOF) error-driven proportional-integral (PI) controllers, which compare the measured joint angles $\left(\theta_{j}, j=1 \ldots 4\right)$ with the reference values 
TABLE I

THE SET OF PARAMETERS USED IN THE SIMULATOR CONTROL. $K_{p}$ : PROPORTIONAL GAIN, $K_{i}$ : INTEGRAL GAIN. THE DEGREES OF FREEDOM ARE: $\theta_{1}$ WRIST FLEXION, $\theta_{2}$ WRIST ULNAR DEVIATION, $\theta_{3}$ FINGER FLEXION, $\theta_{4}$ THUMB FLEXION, $\vartheta_{5}$ GRIP FORCE.

\begin{tabular}{llccccc}
\hline \multicolumn{2}{l}{ Degree of freedom } & $\theta_{1}$ & $\theta_{2}$ & $\theta_{3}$ & $\theta_{4}$ & $\vartheta_{5}$ \\
\hline \multirow{2}{*}{ Action based } & $K_{p}(\mathrm{~N} . \mathrm{cm} / \mathrm{deg})$ & 5 & 5 & 5 & 5 & $20^{*}$ \\
& $K_{i}(\mathrm{~N} . \mathrm{cm} / \mathrm{s} . \mathrm{deg})$ & 2 & 2 & 2 & 2 & $8^{*}$ \\
\hline \multirow{2}{*}{ Optimal } & $K_{p}(\mathrm{~N} . \mathrm{cm} / \mathrm{deg})$ & 13.2 & 13.2 & 6 & 2 & - \\
& $K_{i}(\mathrm{~N} . \mathrm{cm} / \mathrm{s.deg})$ & 5.3 & 5.3 & 2.4 & 0.8 & - \\
\hline \multirow{2}{*}{ * Units are N/Pa }
\end{tabular}

$\left(\theta_{j, r e f}\right)$, and generates reference joint moments $\left(M_{j, r e f}\right)$ to follow the trajectories. The manually tuned controller gains are reported in Table I.

2) Load distribution algorithm: The reference joint moments are translated into tendon tension commands via a load distribution algorithm. Two types of algorithms are considered in this mid-level controller: an optimal controller, and an action-based controller. The optimal controller calculates the minimal tendon loads that produce the required joint moments by considering their strengths and moment arms; the actionbased controller, loads all the tendons that are agonist to a DOF's desired motion independent of their strengths.

a) The optimal controller: Due to the dynamic redundancy in the human musculature, solving for the unique set of tendon tensions requires an additional criterion. This is often the minimization of an effort index. In this controller, the effort index to be minimized is the sum of squared muscle activations [23]:

$$
J=\sum_{i \in \mathcal{M}}\left(\frac{F_{i, r e f}}{F_{i, \max }}-\rho\right)^{2}
$$

The optimization algorithms finds forces $F_{i, r e f}$ that minimize $J$, while considering the constraint:

$$
\begin{gathered}
M_{j, r e f}=\sum_{i \in \mathcal{M}} r_{i, j} \cdot F_{i, r e f}, j \in \mathcal{D} \\
0 \leq F_{i, r e f} \leq F_{i, \max }
\end{gathered}
$$

In (1), the summation is taken over the set of active muscles $\mathcal{M}$. If a muscle is included in $\mathcal{M}$, it is loaded by the controller; otherwise, it will not be loaded $\left(F_{i, r e f}=0\right.$ for $\left.i \notin \mathcal{M}\right)$. The tendon forces are normalized to the maximum muscle forces to represent activations of the muscles. A muscle's maximum force $F_{i, \max }$ is calculated as the product of its physiological cross sectional area (PCSA, from [10]) and the specific muscle tension $\left(\sigma=55 \mathrm{~N} / \mathrm{cm}^{2}\right.$, [24]). The variable $0<\rho<1$ is introduced in the cost function as a co-contraction term [7], shifting the optimal forces to be close to $\rho$ (instead of zero in the non-co-contracting case). $\rho=0$ was used in the presented results. The constraint (2) makes sure that the tendon forces produce the reference joint moments. Here, the variable $r_{i, j}$ is the moment arm of the $i^{\text {th }}$ tendon across the $j^{\text {th }}$ DOF, and $\mathcal{D}$ is the set of controlled DOFs. The moment arms were estimated using the rate of change of tendon excursion with respect to a DOF's rotation $\left(r_{i, j}=\frac{\partial l_{i}}{\partial \theta_{j}}\right.$, [5]).
The optimization problem (1)-(3) can be re-formulated as the following quadratic programming problem:

$$
\begin{gathered}
J=\frac{1}{2} \mathbf{F}^{T} \mathbf{Q F}+\mathbf{C}^{T} \mathbf{F} \\
\mathbf{R F}=\mathbf{M} \\
\mathbf{A F}<\mathbf{b}
\end{gathered}
$$

where $\mathbf{F}$ is the vector containing the tendon forces to be calculated, $\mathbf{M}$ is the vector containing joint moments, $\mathbf{R}$ is the moment arm matrix. $\mathbf{Q}$ is a diagonal matrix, constructed with diagonal elements being $2 / F_{i \text {, max }}^{2}$, and $\mathbf{C}$ is a vector containing $-2 \rho / F_{i, \max }$. Lastly, the inequality constraint (3) is rewritten in matrix form in (6) with the matrices $\mathbf{A}$ and $\mathbf{b}$ defined as:

$$
\mathbf{A}=\left[\begin{array}{cc}
I & {[0]} \\
{[0]} & -I
\end{array}\right], \quad \mathbf{b}=\left[\begin{array}{c}
{\left[F_{i, \max }\right]^{T}} \\
{[0]}
\end{array}\right]
$$

The solution of this quadratic programming is fast and can be implemented for real-time control. A variant of this controller has already been used for the control of a twodegrees-of-freedom wrist simulator [7].

b) The action-based controller: This controller follows the formulation proposed in [25]; each muscle tension is calculated as:

$$
F_{i, r e f}=\sum_{j \in \mathcal{D}} \operatorname{sgn}\left(r_{i, j}\right) \frac{M_{j, r e f}}{\bar{r}}
$$

where the summation is taken over the set of controlled DOFs, $\mathcal{D}$. The joint moments are divided by a representative moment $\operatorname{arm}(\bar{r}$, taken to be $1 \mathrm{~cm})$. The function $\operatorname{sgn}(\cdot)$ is the signum function to which a dead zone is introduced to prevent a muscle from being activated if its moment arm is small (an arbitrarily chosen small threshold), and is defined as:

$$
\operatorname{sgn}(r)=\left\{\begin{array}{lc}
1 & r>1.5 \mathrm{~mm} \\
0 & -1.5 \mathrm{~mm} \leq r \leq 1.5 \mathrm{~mm} \\
-1 & r<-1.5 \mathrm{~mm}
\end{array}\right.
$$

Next, to ensure that the calculated forces are physiological, the following is considered:

$$
F_{i, r e f}=\left\{\begin{array}{lc}
0 & F_{i, r e f}<0 \text { or } i \notin \mathcal{M} \\
F_{i, r e f} & 0 \leq F_{i, r e f} \leq F_{i, \max } \\
F_{i, \max } & F_{i, \max }<F_{i, r e f}
\end{array}\right.
$$

c) Control of grip strength: A modified version of the action-based controller was to implement a feedback loop to control the grip force. For this purpose, an air-filled soft rubber ball (approximately $8 \mathrm{~cm}$ diameter) was attached to a pressure sensor (24P series 5 psi, Honeywell Inc. Morris Plains, NJ, USA) via a flexible hose, to measure the elevation of the pressure as a result of the grip. This increase in pressure served as an indicator of grip strength. For comparison, a light grip by the healthy experimenter (as in holding bottle of water) corresponded to 3-5 $\mathrm{kPa}$ increase in gauge pressure, while a tight grasp increased it to $20-30 \mathrm{kPa}$.

To control the grip with the action-based controller, the measured pressure, $\vartheta_{5}$, was considered as an extra degree 
of freedom and was included in the set of controlled DOFs (i.e., $\vartheta_{5} \in \mathcal{D}$ in (8)). For consistency across all DOFs in (8), the output of the high-level kinematic controller for this DOF was considered to be a generalized moment (actual unit $\mathrm{N}$ ). The generalized moment arms acting on this extra DOF were positive for the finger and thumb flexors $(\operatorname{sgn}(r)=+1)$ and negative for the extensors $(\operatorname{sgn}(r)=-1)$. For other muscles $\operatorname{sgn}(r)=0$. Since the muscles are activated based on their actions (i.e., based on $\operatorname{sgn}(r)$ ), accurate moment arm values were unnecessary. Furthermore, $\bar{r}=1$ (dimensionless) for this DOF.

3) Force controller: The reference tendon forces, determined from either of the aforementioned load distribution algorithms, were applied to the tendons via servo motors. To produce the motor velocity commands, a low-level force controller was implemented. Force feedback was used to calculate the error between the reference and actual forces, which were then fed to a proportional gain $\left(K_{p}=0.106\right.$ $\mathrm{cm} / \mathrm{sN}$ ) to calculate the motor velocity command.

4) Controller implementation: Load cell data acquisition and hardware control were done with a USB-connected National Instruments CompactDAQ Chassis (cDAQ-9178) connected to a laptop computer. The load cell data were acquired as analogue signals (through a full Wheatstone bridge using NI-9237) with approximate sampling rate of $1800 \mathrm{~Hz}$. The low-level force controller update rate was $1000 \mathrm{~Hz}$, and the commands were sent to the motors as analogue voltages (converted to $0-5 \mathrm{~V}$, generated by NI-9264) approximately every $9 \mathrm{~ms}(110 \mathrm{~Hz})$. The kinematics (joint angles from the Qualisys Track Manager software) were streamed at $50 \mathrm{~Hz}$ into LabVIEW. The kinematic controller and load distribution algorithms also updated at $50 \mathrm{~Hz}$.

\section{METHODS: EXPERIMENTS AND DATA ANALYSIS}

\section{A. Specimen preparation}

Fifteen cadaveric arm specimens (truncated from midhumerus) were tested in this simulator. The Tissue Management Committee of the Imperial College Healthcare Tissue Bank approved the use of the specimens for this study according to the Human Tissue Act. To be used on this simulator, a cadaveric arm specimen was stripped of all the soft tissue proximal to mid-forearm (except the elbow joint and proximal radioulnar joint capsule and ligaments), facilitating the access to the extrinsic tendons of the hand. The actuated muscles in our studies were the ones that only affect the wrist: flexor carpi ulnaris (FCU), flexor carpi radialis (FCR), extensor carpi ulnaris (ECU), extensor carpi radialis longus and brevis (ECRL and ECRB), as well as finger and thumb muscles: flexor digitorum superficialis and profundus (FDS and FDP), extensor digitorum communis (EDC), abductor pollicis longus (APL), flexor pollicis longus (FPL), and extensor pollicis longus (EPL). These muscles were chosen in this experiment as the most influential extrinsic muscles of the hand. The omitted extrinsic muscles are small [10] compared to the rest. Surgical sutures (W4843 ETHIBOND EXCEL ${ }^{\mathrm{TM}}$ polyester sutures, Ethicon Inc. Cincinnati, OH, USA) were stitched to the tendons using Krackow suture technique to provide a strong interface between the tendons and the steel wires. The forearm was held in a neutral supination/pronation position using a $2 \mathrm{~mm}$ Kirschner wire, fixating the radius to the ulna at mid-shaft height. Finally, the arm was secured to the setup in a vertical orientation using three external fixation pins (Hoffmann 3, Stryker, Kalamazoo, MI, USA) that were drilled into the proximal half of the ulna.

\section{B. Experimental trials}

Six cycles of various hand movements such as wrist flexion/extension, dart-throwing motion [26], and opening/closing of the hand were performed with each specimen (see the supplementary material for a list of all 117 trials tested on each specimen). To showcase the capabilities of the simulator, two example trials are presented and discussed: opening and closing of the hand, and controlled power-grip. However, the performance of the controllers (repeatability, tracking error, and resulting tendon forces, see below) was evaluated considering all applicable tested trials.

\section{Data analysis}

The first cycle in each trial was discarded to remove any transient response, and the remaining five cycles were analyzed. The data in each cycle was time-normalized $(\tau \in[0,1])$, and re-sampled to 500 time steps.

Because of the skewed distributions of our variables, median and the robust estimator MAD (median absolute deviation) [27], [28] were used to analyze the results. MAD is defined as

$$
\operatorname{MAD}(x)=b \underset{i}{\operatorname{Med}}\left(\left|x_{i}-\underset{j}{\operatorname{Med}}\left(x_{j}\right)\right|\right),
$$

where $\operatorname{Med}_{i}$ is the median taken over the indices $i$, and $b=$ 1.4826 .

Repeatability of the simulations was evaluated using the variations in the kinematics and tendon forces. To define kinematic repeatability, the MAD of the measured kinematics was calculated across the five cycles of a trial in each time step, resulting in a trajectory of inter-cycle dispersity:

$$
\rho_{j}^{l}[\tau]=\underset{k}{\operatorname{MAD}}\left(\theta_{j}^{l(k)}[\tau]\right), \quad k=1 \ldots 5
$$

with $\theta_{j}^{l(k)}[\tau]$ begin the measured value of the $j^{t h}$ DOF in $k^{t h}$ cycle of trial $l$ at time step $\tau$. The median of this trajectory $\left(\widetilde{\rho}_{j}^{l}=\operatorname{Med}_{\tau}\left(\rho_{j}^{l}[\tau]\right)\right)$ was calculated over the course of the movement to result in a single repeatability value for DOF $j$ in trial $l$. The distribution of $\widetilde{\rho}_{j}^{l}$ across the tests trials was used to infer kinematic repeatability for each DOF. Similarly, for the dynamic repeatability, the median of the MAD-trajectory of the tendon forces was used.

The performance of the control algorithms was evaluated in terms of the kinematic error and tendon forces. In each cycle of a movement, the median kinematic error (difference between the reference and measured kinematics) for each controlled DOF across the duration of cycle was calculated as:

$$
{\widetilde{e_{j}}}^{l(k)}=\underset{\tau}{\operatorname{Med}}\left(\theta_{j}^{l(k)}[\tau]-\theta_{j, r e f}[\tau]\right), \quad \tau \in[0,1]
$$


To analyze the forces, the median total force and median total squared force were calculated for each cycle, i.e.:

$$
\begin{array}{r}
\widetilde{F}^{l(k)}=\underset{\tau}{\operatorname{Med}}\left(\sum_{i \in \mathcal{M}} F_{i}^{l(k)}[\tau]\right), \quad \tau \in[0,1] \\
{\widetilde{F^{2}}}^{l(k)}=\operatorname{Med}_{\tau}\left(\sum_{i \in \mathcal{M}}\left(F_{i}^{l(k)}[\tau]\right)^{2}\right), \quad \tau \in[0,1]
\end{array}
$$

The distributions of $\widetilde{e_{j}}, \widetilde{F}$, and $\widetilde{F^{2}}$ over all cycles/trials were analyzed.

To statistically compare the repeatability and performance of the two control algorithms, non-parametric Mann-Whitney Utests were performed, with a statistical significance threshold of $p=0.05$.

\section{RESUlTS}

\section{A. Force tracking of the low-level force controller}

The low-level force controller adjusted the velocities of the actuators to make sure that they tensioned the tendons according the specified reference values (outputted by the load distribution algorithm). The error between the measured and the reference force values across all trials and muscles had a median of $1.52 \mathrm{~N}(\mathrm{MAD}=1.82 \mathrm{~N})$ with distributions shown in Fig. 2a. Not all muscles exhibited similar performance. An example trial (Fig. 2b) shows that flexor carpi ulnaris (FCU) was overloaded during the extension phase, and fell behind during flexion. A reverse effect is visible for extensor carpi radialis $(\mathrm{ECRL} / \mathrm{ECRB})$.

\section{B. Repeatability}

Across all tested trials, the median error between individual cycle durations and the reference duration was $0.04 \mathrm{~ms}$ (MAD $=2.3 \mathrm{~ms}$ ) with the action based controller, and $0.03 \mathrm{~ms}$ (MAD $=2.3 \mathrm{~ms}$ ) with the optimal controller. Statistically, the duration error with either of the controllers could not be differentiated from zero $(p>0.78)$, or from each other $(p=0.81)$.

The median kinematic repeatability across all trials was better than $0.17^{\circ}$ for both control algorithms in all DOFs (Fig. 3a). Statistical tests show significant differences in all DOFs between the two controllers $(p<0.05)$.

The median dynamic repeatability over all trials was at most $0.25 \mathrm{~N}$ among all muscle using either controllers (Fig. 3b). Except for FCU, FCR, and ECRB, the controllers resulted in statistically different dynamic repeatability distributions $(p<$ $0.05)$.

\section{Controller performance}

The two controllers resulted in comparable kinematic tracking performance; the action-based controller generally resulted in lower error for the wrist motion, while the optimal controller controlled the fingers and thumb more closely (Fig. 4). For all four DOFs, the difference between the kinematic error resulting from the two controllers was statistically significant $(p<0.05)$.

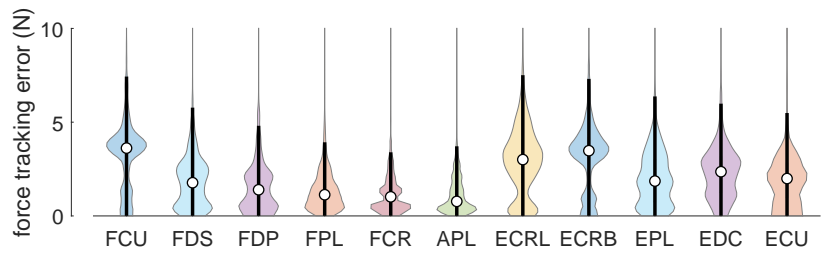

(a)

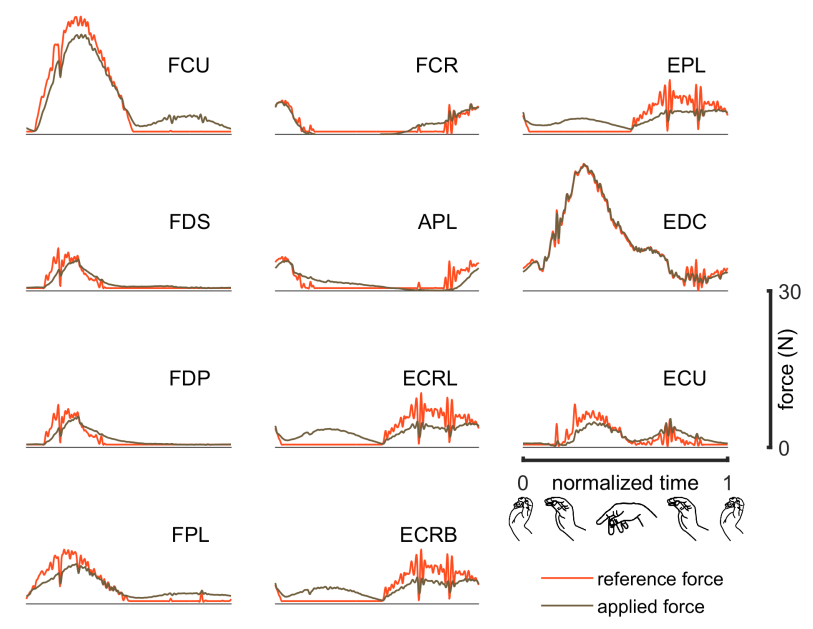

(b)

Fig. 2. Tracking performance of the low-level force controller for the linear actuators. (a) Violin plots of the error between the reference and actual tendon tensions. All trials from both controllers are pooled together. Black bars show the 1st and 3rd quartiles, white dots indicate the median, and the shaded areas show of the probability distributions. (b) An example of the reference tension trajectories and the actual force applied to the tendons during a dart-throwing motion using the action-based controller. In this motion, wrist starts from $30^{\circ}$ extension with $10^{\circ}$ radial deviation, moves to $30^{\circ}$ flexion with $10^{\circ}$ ulnar deviation, and then returns.

The optimal controller on average controlled the kinematics with lower tendon forces (Fig. 5). $\widetilde{F}$ had a median of $45.4 \mathrm{~N}$ $(\mathrm{MAD}=13.9 \mathrm{~N})$ and $54.0 \mathrm{~N}(\mathrm{MAD}=16.4 \mathrm{~N})$ for the optimal and action-based controllers, respectively $(p<0.05)$, when taken across all tested trials. Likewise $\widetilde{F^{2}}$ had a median of $392.0 \mathrm{~N}^{2}$ $\left(\mathrm{MAD}=258 \mathrm{~N}^{2}\right)$ with the optimal controller and $451.1 \mathrm{~N}^{2}$ $\left(\mathrm{MAD}=305 \mathrm{~N}^{2}\right)$ with the action-based controller $(p<0.05)$. Between-controller comparison of the individual trials also showed that the total forces with the optimal controller were smaller than those of the action-based controller (median difference $=7.9 \mathrm{~N}, \mathrm{MAD}=8.1 \mathrm{~N}, p<0.05)$.

\section{Simulation of grasp}

An advantage of the developed simulator is its ability to move digits independently of the wrist for the simulation of grasping function. During opening of the hand while actively keeping the wrist steady (Fig. 6), forces by EDC and EPL are counter-balanced by FCU. The unopposed flexion of the fingers/thumb requires less force than extension (Fig. 6), due to the resting length of the hand musculotendon units that corresponds to a half-closed posture.

The pressure inside the rubber ball was controlled successfully by the specimen in a power-grip simulation (Fig. 7), with median(MAD) tracking error of $0.21(0.11) \mathrm{kPa}$. 

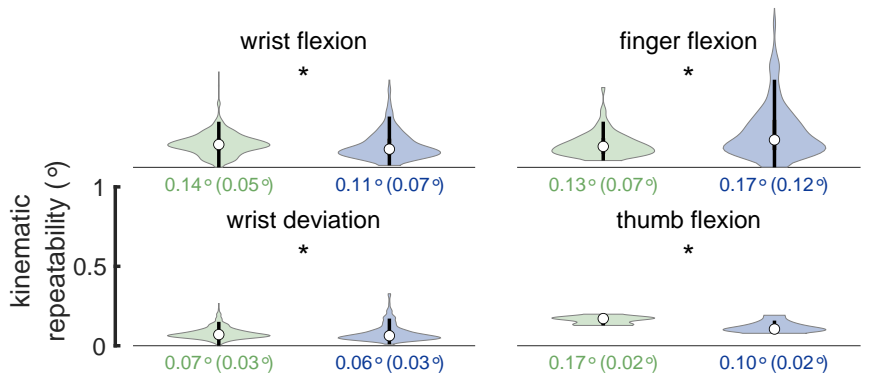

(a)
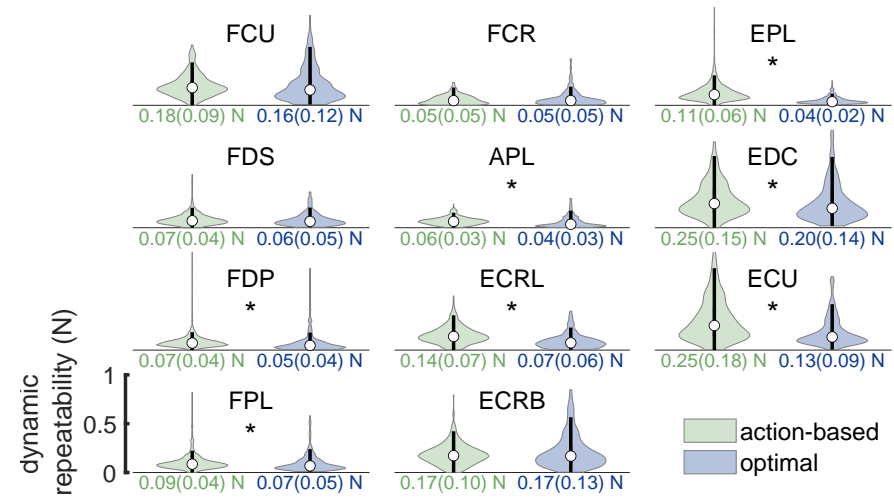

(b)

Fig. 3. Violin plots of (a) kinematic repeatability and (b) dynamic repeatability. Asterisks $(*)$ show statistically significant difference between the two controllers $(p<0.05)$. Numbers below the plots indicate median (MAD) repeatability.

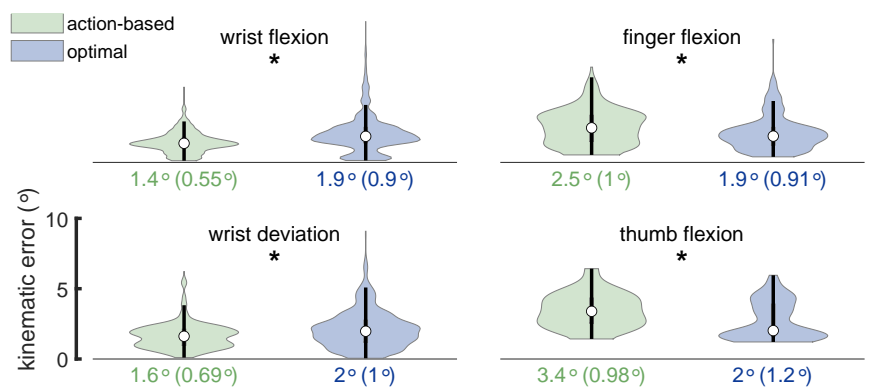

Fig. 4. The violin plots of the kinematic error, resulting from the two load distribution algorithms. Asterisk (*) represents statistically different $(p<0.05)$ distributions of kinematic error. Numbers below the plots indicate median (MAD) error.

\section{DiscusSION}

This paper presented the design and control of a novel in vitro hand and wrist simulator. The experimental results verify our primary design objective of recreating various wrist, finger, and thumb movements, by actuating the extrinsic muscles of the hand. Despite the added complexity due to the coupled wrist and fingers dynamics, the wrist kinematic error (median $<1.6^{\circ}$, Fig. 4 ) and the dynamic repeatability (median $<0.25 \mathrm{~N}$, Fig. 3b) were better than the previous state-of-theart wrist-only simulator (kinematic error $<1.8^{\circ}$, repeatability $<1 \mathrm{~N}$ [29]). This hand simulator was also designed to be versatile, as each DOF could be controlled independently, or left uncontrolled. For instance, one can control the wrist while allowing the digits to move freely according to their dynamics.

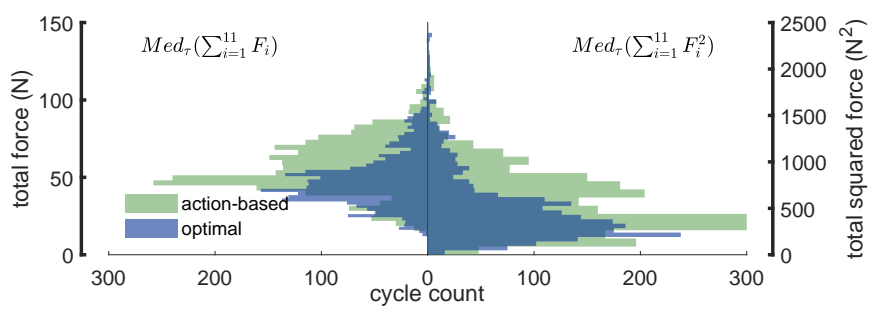

Fig. 5. Comparison of the forces resulting from the two control algorithms. All cycles of all trials were included in the histograms.

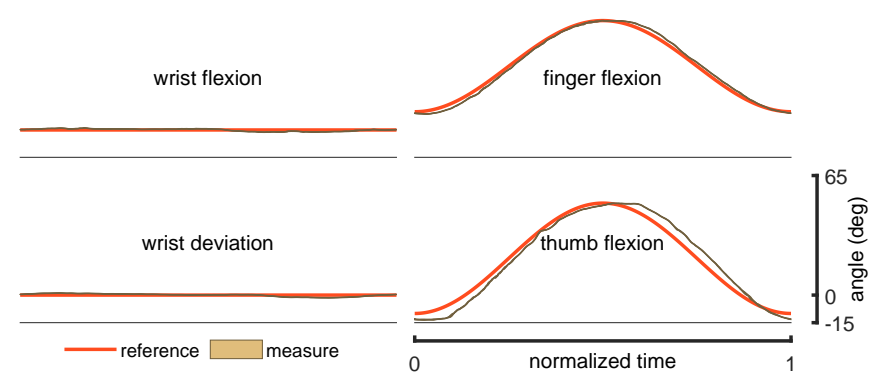

(a)

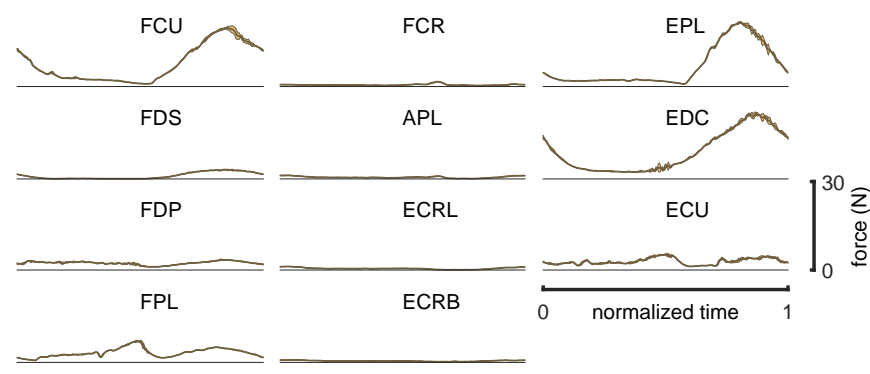

(b)

Fig. 6. An example simulation of hand grasp using the action-based algorithm. (a) The provided reference and tracked kinematics for the degrees of freedom; (b) The tendon tensions that created the movement. Shaded bands show median \pm MAD for five cycles of this motion.

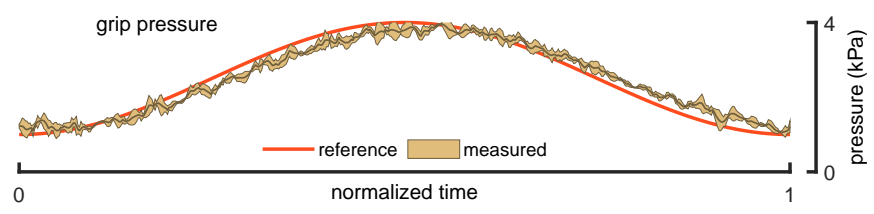

(a)

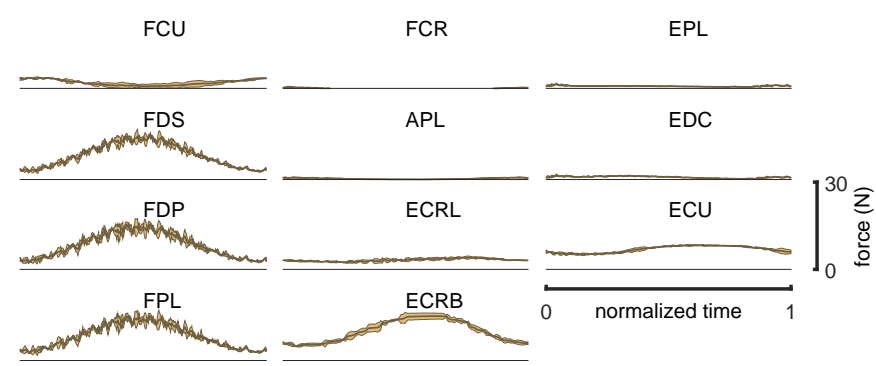

(b)

Fig. 7. A representative example for the simulation of grip using the actionbased algorithm. (a) The reference and tracked grip pressure trajectories; (b) The corresponding tendon tensions. Shaded bands show median \pm MAD for five cycles of this motion. 
This selective control can be achieved by including the needed DOFs in set $\mathcal{D}$ in (2), or (8). Moreover, any of the muscles can be turned on or off by choice by appropriately selecting the set $\mathcal{M}$.

The developed feedback controller ensures that the specimen follows the reference kinematics by controlling the tendon tensions. This is in contrast to excursion-based simulator controllers, in which the low-level controller is omitted, and the actuators' velocity commands are calculated directly using feedback from kinematic measurements [1], [6]. In excursionbased controllers, movements of agonist actuators are often calculated via activation ratios, which is the relative ratio of their tendon moment arms to that of a prime mover (e.g. [2], [7]). If there is mismatch between the estimated and real moment arms, a tendon may be overloaded or go slack. From a tendon excursion point of view, the human musculoskeletal system is in fact over-determined [30] - there is one and only one solution that satisfies the constraints of tendon excursions. In this case, the tendon loadings depend on the accuracy of the moment arm ratios. The force-based control scheme that is used in this simulator has the important advantage that the tendon tensions can be independently specified, and the motors pull the tendons just enough to produce the needed tension.

The results highlight the similarities and difference between the two control algorithms. Firstly, both algorithms controlled the coupled dynamics of the wrist, fingers, and thumb with high repeatability (expected trial-to-trial variability of $<.25$ $\mathrm{N}$ and $<.2^{\circ}$ ). Kinematic tracking of the two methods was also high, although, across multiple trials, the action-based controller gave tighter wrist kinematic tracking, while the optimal one resulted in smaller tracking errors for the finger and thumb. Though the two controllers resulted in repeatability and kinematic errors that were statistically different, the absolute values of these differences were small.

The meaningful difference between the two controllers was in the magnitude of tendon forces required to perform the movements. As expected, the optimal controller resulted in significantly lower forces. The sum of the squared tendon tensions (quantity that optimization algorithm minimizes) from the optimal controller was $13 \%$ lower than that from the action-based controller. The summation of all tendon forces was also lower with the optimal controller (16\% lower than the action-based). Consequently, the optimal controller better represented the optimality principle (minimal muscular effort) that is thought to be the main principle underlying the neural control of movements [18]. However, despite its greater biofidelity, the optimal controller was more susceptible to occasional oscillations, as also reported in previous simulators [7]. We speculate that these oscillations may be attributed to the need for additional computational time due to the optimization, which ultimately affects the controller cycle time and consequently results in inferior force control performance. It is well-discussed in the literature that delays greatly reduce the control bandwidth in force-reflecting haptic systems [31].

The resulting muscle forces from both of the two controllers are lower than previously reported forces in wrist simulators [1]-[4]. One major contributor to the lower forces is the inclusion of more muscles for the control of the movements.
Furthermore, the level of unwanted co-contraction is lower. An example of such unwanted co-contractions is visible in ECRL, ECRB, and EPL during the flexion phase of the dart throwing motion in Fig. 2b. Due to the limited travel velocity of the linear actuators, the low-level force controller failed to off-load the tendons fast enough, resulting in unplanned antagonistic tensions. This issue largely has been mitigated in our simulator by incorporating a force-controlled scheme for more accurate loading of tendons, as well as increasing the controller update rate, which lowers the time delays and allows for larger control gains without losing stability. Due to the difficulty of measuring tendon forces in vivo, it remains to be investigated how close the simulated forces are to those in natural human movements.

This in vitro simulator has been developed primarily for hand surgery studies, where the effects of surgical reconstructions on hand kinematics and muscle forces are investigated. To reliably isolate and analyze effects of interventions, it is necessary to remove any external random effect due to variability in kinematics or other test conditions. The designed controllers recreate human-like movements and guarantee repeatable and accurate movements, thus enabling researchers to study surgical procedures. Additionally, the implications of the results obtained from this simulator go beyond surgical applications. For instance, the tendon forces in combination with the measured hand kinematics are invaluable resource in validating in silico simulation results. By including detailed muscle models in the simulation, it is possible to separate the contributions of the active force production from the passive properties of the muscle, which has direct implications in human motor control studies.

Although this hand simulator is a major improvement to existing wrist simulators, to fully implement hand functionality, the intrinsic muscles (which have their origins and insertions within the hand) must be considered. For instance, extension of the fingers is a complex motion, shared by extensor digitorum communis (EDC) which inserts at the base of the middle phalanx, and the intrinsic interossei and lumbricals which insert at the base of the distal phalanx. Motorization of the EDC only causes a predictable clawing pattern, with the metacarpophalangeal joints hyperextending, and the interphalangeal joints remaining semi-flexed. Furthermore, due to the limited number of actuators, the four branches of FDS, FDP, and EDC could not be moved independently. Four extrinsic muscles (palmaris longus, extensor digiti minimi, extensor indicis proprius, and extensor pollicis brevis) were also omitted in this simulator for the same reason. These muscles have been known to have a limited effect on hand function; in addition to having the smallest force production capacity, they are often used for tendon graft or tendon transfer surgery [32] because their actions are mostly covered by other muscles.

\section{CONCLUSION}

An advanced physiological hand simulator and two feedback control algorithms to generate accurate movements in hand specimens has been presented. This simulator is the first of its kind that can control movements of the wrist, 
fingers, and thumb by actuating all the major extrinsic hand muscles. This simulator was force-controlled, meaning the tendons could move independently, and hence the controller was more robust to errors in estimating tendon moment arms. Both control methods were successful in accurately controlling the movements (median kinematic error $<3.4^{\circ}$ in all DOFs). The optimal controller employed smaller total forces, while maintaining comparable tracking accuracy. Control of grip force is another novel feature of this simulator, where an indicator of grip force was used to construct the feedback control loop. As this simulator is better able to mimic physiological actuation of the linked DOFs in the human hand, it can be a viable tool in biomechanical studies, analysis of the effects of surgical interventions on kinematics/kinetics, and testing of movement related deficits.

\section{ACKNOWLEDGMENT}

This research was supported by grants from the Engineering and Physical Sciences Research Council (EP/R020809/1) and the British Society for Surgery of the Hand. The authors wish to thank Dr. Farokh Atashzar and Mr. Grey Giddins for their valuable discussions.

\section{REFERENCES}

[1] F. W. Werner, A. K. Palmer, J. H. Somerset, J. J. Tong, D. B. Gillison, M. D. Fortino, and W. H. Short, "Wrist joint motion simulator," Journal of Orthopaedic Research, vol. 14, no. 4, pp. 639-646, 1996.

[2] L. Leonard, D. M. Sirkett, I. J. Langdon, G. Mullineux, D. G. Tilley, P. S. Keogh, J. L. Cunningham, M. O. T. Cole, P. H. Prest, G. E. B. Giddins, and A. W. Miles, "Engineering a new wrist joint replacement prosthesis - A multidisciplinary approach," Proceedings of the Institution of Mechanical Engineers, Part B: Journal of Engineering Manufacture, vol. 216, no. 9, pp. 1297-1302, 2002.

[3] S. Erhart, M. Lutz, R. Arora, and W. Schmoelz, "Measurement of intraarticular wrist joint biomechanics with a force controlled system," Medical Engineering and Physics, vol. 34, no. 7, pp. 900-905, 2012.

[4] D. S. Shah, C. Middleton, S. Gurdezi, M. D. Horwitz, and A. E. Kedgley, "The effects of wrist motion and hand orientation on muscle forces: A physiologic wrist simulator study," Journal of Biomechanics, vol. 60, pp. 232-237, 2017.

[5] K. N. An, Y. Ueba, E. Y. Chao, W. P. Cooney, and R. L. Linscheid, "Tendon excursion and moment arm of index finger muscles," Journal of Biomechanics, vol. 16, no. 6, pp. 419-425, 1983.

[6] D. S. Shah, C. Middleton, S. Gurdezi, M. D. Horwitz, and A. E. Kedgley, "The importance of abductor pollicis longus in wrist motions: A physiological wrist simulator study," Journal of Biomechanics, vol. 77, pp. 218-222, 2018.

[7] D. S. Shah and A. E. Kedgley, "Control of a wrist joint motion simulator: A phantom study," Journal of Biomechanics, vol. 49, no. 13, pp. 30613068, 2016.

[8] F.-C. Su, Y. Chou, C. Yang, G. Lin, and K. An, "Movement of finger joints induced by synergistic wrist motion," Clinical Biomechanics, vol. 20, no. 5, pp. 491-497, 2005.

[9] S. V. Gehrmann, R. A. Kaufmann, and Z. M. Li, "Wrist Circumduction Reduced by Finger Constraints," Journal of Hand Surgery, vol. 33, no. 8, pp. $1287-1292,2008$

[10] K. R. S. Holzbaur, W. M. Murray, G. E. Gold, and S. L. Delp, "Upper limb muscle volumes in adult subjects," Journal of Biomechanics, vol. 40, no. 4, pp. 742-749, 2007.

[11] Jin Bo Tang, J. Ryu, V. Kish, and S. Wearden, "Effect of radial shortening on muscle length and moment arms of the wrist flexors and extensors," Journal of Orthopaedic Research, vol. 15, no. 3, pp. 324 330, 1997.

[12] K. D. Han, J. M. Kim, M. V. DeFazio, R. J. Bello, R. D. Katz, B. G. Parks, and K. R. Means, "Changes in Wrist Motion After Simulated Scapholunate Arthrodesis: A Cadaveric Study," Journal of Hand Surgery, vol. 41, no. 9, pp. e285-e293, 2016.
[13] M. M. León-Lopez, G. Salvà-Coll, M. Garcia-Elias, A. Lluch-Bergadà, and M. Llusá-Pérez, "Role of the extensor carpi ulnaris in the stabilization of the lunotriquetral joint. An experimental study," Journal of Hand Therapy, vol. 26, no. 4, pp. 312-317, 2013.

[14] K. Pervaiz, W. H. Bowers, J. E. Isaacs, J. R. Owen, and J. S. Wayne, "Range of Motion Effects of Distal Pole Scaphoid Excision and Triquetral Excision After Radioscapholunate Fusion: A Cadaver Study," Journal of Hand Surgery, vol. 34, no. 5, pp. 832-837, 2009.

[15] H. Park and D. M. Durand, "Motion control of musculoskeletal systems with redundancy.", Biological Cybernetics, vol. 99, no. 6, pp. 503-16, 2008.

[16] D. Blana, R. F. Kirsch, and E. K. Chadwick, "Combined feedforward and feedback control of a redundant, nonlinear, dynamic musculoskeletal system," Medical \& Biological Engineering \& Computing, vol. 47, pp. 533-542, apr 2009.

[17] N. Mehrabi, R. Sharif Razavian, B. Ghannadi, and J. McPhee, "Predictive simulation of reaching moving targets using nonlinear model predictive control," Frontiers in Computational Neuroscience, vol. 10 , no. 143, 2017.

[18] E. Todorov and M. I. Jordan, "Optimal feedback control as a theory of motor coordination," Nature Neuroscience, vol. 5, no. 11, pp. 1226$1235,2002$.

[19] D. Liu and E. Todorov, "Hierarchical optimal control of a 7-DOF arm model," in 2009 IEEE Symposium on Adaptive Dynamic Programming and Reinforcement Learning, no. 2, (Nashville, TN, USA), pp. 50-57, IEEE, 2009.

[20] K. C. D. Fu, F. D. Libera, and H. Ishiguro, "Extracting motor synergies from random movements for low-dimensional task-space control of musculoskeletal robots," Bioinspiration \& Biomimetics, vol. 10, no. 5, p. 056016, 2015.

[21] R. Sharif Razavian, B. Ghannadi, N. Mehrabi, M. Charlet, and J. McPhee, "Feedback Control of Functional Electrical Stimulation for 2-D Arm Reaching Movements," IEEE Transactions on Neural Systems and Rehabilitation Engineering, vol. 26, no. 10, pp. 2033-2043, 2018.

[22] G. Wu, F. C. van der Helm, H. (DirkJan) Veeger, M. Makhsous, P. Van Roy, C. Anglin, J. Nagels, A. R. Karduna, K. McQuade, X. Wang, F. W. Werner, and B. Buchholz, "ISB recommendation on definitions of joint coordinate systems of various joints for the reporting of human joint motion-Part II: shoulder, elbow, wrist and hand," Journal of Biomechanics, vol. 38, pp. 981-992, may 2005.

[23] A. Erdemir, S. McLean, W. Herzog, and A. J. van den Bogert, "Modelbased estimation of muscle forces exerted during movements," Clinical Biomechanics (Bristol, Avon), vol. 22, no. 2, pp. 131-154, 2007.

[24] T. D. O'Brien, N. D. Reeves, V. Baltzopoulos, D. A. Jones, and C. N. Maganaris, "In vivo measurements of muscle specific tension in adults and children," Experimental Physiology, vol. 95, no. 1, pp. 202-210, 2010.

[25] K. M. Jagodnik, D. Blana, A. J. van den Bogert, and R. F. Kirsch, "An optimized proportional-derivative controller for the human upper extremity with gravity," Journal of Biomechanics, vol. 48, no. 13, pp. 3701-3709, 2015

[26] H. Moritomo, E. P. Apergis, G. Herzberg, F. W. Werner, S. W. Wolfe, and M. Garcia-Elias, "2007 IFSSH Committee Report of Wrist Biomechanics Committee: Biomechanics of the So-Called Dart-Throwing Motion of the Wrist," Journal of Hand Surgery, vol. 32, no. 9, pp. 1447-1453, 2007.

[27] C. Leys, C. Ley, O. Klein, P. Bernard, and L. Licata, "Detecting outliers: Do not use standard deviation around the mean, use absolute deviation around the median," Journal of Experimental Social Psychology, vol. 49, no. 4, pp. 764-766, 2013.

[28] P. J. Rousseeuw and C. Croux, "Alternatives to the Median Absolute Deviation," Journal of the American Statistical Association, vol. 88, pp. 1273-1283, dec 1993.

[29] D. S. Shah, Design and Implementation of a Physiological Wrist Simulator with Applications to Surgical Reconstructions. Phd thesis, Imperial College London, 2017.

[30] A. Marjaninejad and F. J. Valero-Cuevas, "Should Anthropomorphic Systems be "Redundant"?," in Biomechanics of Anthropomorphic Systems, vol. 124, pp. 7-34, Springer International Publishing, 2019.

[31] R. Anderson and M. Spong, "Bilateral control of teleoperators with time delay," IEEE Transactions on Automatic Control, vol. 34, no. 5, pp. 494$501,1989$.

[32] F. Moutet, D. Corcella, A. Forli, M. Bouyer, M. Aribert, and J.-P. Giot, "General principles of tendon transfers: The ten commandments," Hand Surgery and Rehabilitation, pp. 1-5, may 2021. 